\title{
DUST TOWARDS THE GALACTIC CENTRE
}

\author{
D. K. AITKEN \\ University College, University of New South Wales, \\ Australian Defense Force Academy, \\ Campbell, ACT 2600, \\ Australia
}

\begin{abstract}
The observed infrared properties of dust extinction along the line of site to the Galactic Centre are compared with those in the local interstellar medium. Further constraints on the nature of the dust grains are provided by polarimetric observations, which also lead to information on the stratification of the material along the line of sight. Finally some implications of the large polarized emission seen from the central regions are discussed.
\end{abstract}

\section{INTRODUCTION}

Unobservable at visible wavelengths, with extinction $A_{v} \simeq 30 \mathrm{mag}$, the Galactic Centre lies along the longest path length in the diffuse interstellar medium (ISM) which can presently be observed in the near and mid infrared. The results of Glass et al. (1987) at J, H, and $\mathrm{K}$ show this extinction dramatically: the central star cluster is not apparent at $\mathrm{J}$ but is prominent at longer wavelengths. Their work also shows that, on a scale of fractions of a degree, there are regions of much larger extinction. Uneven extinction has also been reported by Lebofsky \& Rieke (1987) with excursions up to $A_{v} \sim 70 \mathrm{mag}$. The clumpiness can be related to intervening molecular clouds along the line of sight since, as noted by Glass and co-workers, it correlates with ${ }^{13} \mathrm{CO}$ emission. On a much smaller scale many molecular species have been observed in a ring of radius $\sim 2 \mathrm{pc}(\sim 50 \mathrm{arcsec})$ surrounding the Centre. However it seems we view Sgr A itself through a window relatively clear of molecular material.

While some less distant infrared sources suffer greater extinction, these are embedded in dense clouds and much of their extinction is local; the grain properties and the physical environment differ from those in the diffuse ISM. Such a long path length through the diffuse ISM as the luminosity of Sgr A makes accessible is exceptional, but the source is unique within the Galaxy and its properties not well defined in terms of familiar objects. Nevertheless, the broad aspects of absorption features at 3.0, 3.4, 9.7 and $19 \mu \mathrm{m}$ are clearly apparent, while at the same time a number of features which are prominent in molecular cloud material are absent (e. g. see the recent reviews by Roche, 1988 and Whittet, 1988). 


\section{THE EXTINCTION CURVE IN THE INFRARED}

\subsection{THE CONTINUUM}

Studies of reddened stars within a few kpc of the solar system determine the extinction curve of the diffuse ISM in the near infrared. Whittet (1988) has presented a recent evaluation of this out to $5 \mu \mathrm{m}$, which is based on data from a variety of sources (Whittet and van Breda, 1980; Castor and Simon, 1983; Leitherer and Wolf, 1984; Savage and Mathis, 1979) involving hundreds of reddened early type stars obscured predominantly by diffuse material. Rieke and Lebofsky (1985) find essentially the same extinction law to a number of discrete sources in the Galactic Centre, and more recently Lebofsky and Rieke (1987), in a study involving $\mathrm{H}$ and $K$ photometry of 1400 stars in the central few arcmin, deduce a mean extinction to the central stellar population of $A_{v}=30$ mag.

\subsection{ABSORPTION FEATURES}

Molecular cloud sources show dust absorption features between 2 and $20 \mu \mathrm{m}$ (e.g. Whittet, 1988; Willner, 1984) many of which can be associated with complex ices existing as mantle materials. However, the only significant features apparent in the diffuse material towards Sgr A are those due to silicates at 9.7 and $19 \mu \mathrm{m}$ and other features at 3.0 and $3.4 \mu \mathrm{m}$. Tapia et al., 1989 have reported these latter two features in the direction of some highly reddencd field stars but otherwise they have not shown up in any other studies of the diffuse ISM, perhaps because of the relatively small optical depths available.

Extension of the extinction curve from the visible to beyond $\sim 5 \mu \mathrm{m}$ and to include the $9.7 \mu \mathrm{m}$ silicate absorption, relies on a few very luminous stars which lie in directions of large extinction, up to $A_{v}=13 \mathrm{mag}$. VI Cyg12 has been used by Gillett et al. (1975) and Rieke (1974) to determine a relation between $A_{v}$ and the silicate optical depth $\tau_{\text {sil }}$. Roche and Aitken (1984) also used VI Cyg12 together with a number of dusty Wolf-Rayet stars to find a good correlation between the visible and $10 \mu \mathrm{m}$ extinction in the local ISM up to a distance of a few kpc with $A_{v} / \tau_{9.7}=18.5 \pm 1.5$. They also found that the emissivity function appropriate to circumstellar shells of late type oxygen-rich supergiants, typified by that of $\mu \mathrm{Cep}$, gave a much better fit to the data than that of the Trapezium region of Orion; this latter in turn gives better fits to the dust in molecular clouds. The difference between these emissivity functions is not merely the presence of a larger proportion of featureless component in molecular clouds; the circumstellar profile is narrower after subtraction of the smooth continuum.

Rieke and Lebofsky (1985) have investigated the interstellar absorption to the Galactic Centre out to the $\mathbf{N}$ band on a number of stellar sources and included narrow band photometry at $10.4 \mu \mathrm{m}$ to obtain information on the silicate feature; these sources are too faint to have been investigated spectroscopically so far in this wavelength region. The ionized regions of Sgr A in the northern arm and east-west bar are very luminous in the infrared, and 8-13 $\mu \mathrm{m}$ spectroscopy has been possible on these (e.g. Becklin et al., 1978; Aitken et al., 1986). It has been established from the observed ratios of Brackett lines and comparison with the freefree radio continuum (Brown et al., 1981; Wade et al., 1987) that the extinction is essentially uniform over these sources with an average value corresponding to $A_{v}$ 
$=27 \mathrm{mag}$, substantially the same as to the stellar cluster. However, here the form of the underlying emission complicates the problem of determining the extinction curve. Acceptable fits to the data using familiar emissivity functions have been found to be possible only using circumstellar shell emissivity ( $\mu \mathrm{Cep}$ ) for extinction and emission, with an additional smooth component (preferentially of form $\lambda^{-2}$ ) in emission (Roche and Aitken, 1985). This procedure is found to be adequate for the prominent mid infrared sources in the Sgr A complex with an optical depth $\tau_{\mu \text { Cep }}=3.6$ at $9.7 \mu \mathrm{m}$; the ratio of the silicate/smooth component is a free parameter. Use of the Trapezium emissivity function in any combination has lead to inadequate fits.

While the preference for the circumstellar shell emissivity accords well with findings for the local ISM, for this path $A_{v} / \tau_{\text {sil }}$ is very much lower at $\simeq 8-9$. Note that this result implies that in the inner regions the ratio visual/silicate extinction must be lower still, assuming that the local component in this direction is "normal". This increase in the ratio of silicate and oxygen-rich grains to the material which absorbs at shorter wavelengths, presumably carbon based, implies an increasing abundance of heavy elements towards the Centre (Greenberg and Hong, 1974; Roche and Aitken, 1985) and corresponds with the well established rarity of carbon stars in the inner regions of the Galaxy (Blanco and McCarthy, 1981, 1983; Fuenmayor, 1981).

There is no sign of ice band absorption at $3.08 \mu \mathrm{m}$ towards the Galactic Centre, nor is this evident in the spectrum of VI Cyg12 (Gillett et al., 1975). A peculiar absorption feature in the near infrared source IRS 19 has been reported by Willner and Pipher (1982) and McFadzean et al. (1988). The only other obvious features seen along the Galactic Centre path are at 3.0 and $3.4 \mu \mathrm{m}$ (Butchart et al., 1986). Although the precise identification of these features is uncertain, they have been attributed to $O-H$ and $C-H$ bonds respectively, and the latter acceptably modelled in terms of a mixture of hydrogenated amorphous carbon (HAC) by Duley and Williams (1983). Both these features are absent in VI Cyg12 at significantly less than the ratio $1 / 3$ implied by the visible extinctions to it and to Sgr $\mathbf{A}$. In view of the enhancement of silicate material to the latter this might appear to support the suggestion that the $3.0 \mu \mathrm{m}$ feature is due to $O H$ bonds in silicates (Duley, 1988) but is seemingly at variance with the $3.4 \mu \mathrm{m}$ feature arising from carbon based material. When observations from ISO and SIRTF become possible it may be possible to address these matters with adequate statistics.

\section{DUST EMISSION}

Silicate emission from the centrally ionized filaments is required to account for their spectral shape between 8-13 $\mu \mathrm{m}$; this has the characteristic wavelength dependance of circumstellar shell emission ( $\mu \mathrm{Cep})$, rather than that of a typical HII region (Trapezium-like emission), and the grains appear to be free of mantles. Far infrared emission from much cooler dust arises from an apparently toroidal structure (Becklin, Gatley and Werner, 1982; Lester et al., 1987; Davidson et al., 1988) which surrounds the Centre and is presumably related to the 2 pc molecular ring, although there appear to be significant structural differences. If the ionized filaments are derived from this molecular ring then their apparently bare grains imply that either the molecular ring is a transient phenomenon or grains spend little time within it. 
There is no sign of the emission features at 8.6 and $11.3 \mu \mathrm{m}$, now frequently attributed to polycyclic aromatic hydrocarbon molecules (PAHs). These features are ubiquitous in the nuclei of "starburst" galaxies (e.g. Roche, 1989), and it is not clear whether their absence in Sgr A is due to the relatively small projected beam size or to the presence of hard radiation from the nucleus, as the features are not seen in active nuclei. However the associated $3.28 \mu \mathrm{m}$ feature has been reported to be widespread throughout Sgr A (Gatley, 1984).

\section{POLARIMETRIC STUDIES}

Dust grains responsible for the interstellar extinction are capable of alignment and polarimetric and spectropolarimetric studies lead to further constraints on the distribution of grains and their physical and chemical properties. While a number of heavily obscured sources in molecular clouds have been studied in this way, the Galactic Centre is the only source against which the diffuse ISM has been studied polarimetrically out to $100 \mu \mathrm{m}$.

In a study of near infrared polarization of stars in the direction of the centre of the Galaxy, Kobayashi et al. (1980) found a fairly uniform amount and position angle of polarization at $2.2 \mu \mathrm{m}$ over a field of $7 \times 7$ arcmin extent. They interpreted the polarization as due to dichroic absorption in a distribution of grains partially aligned by a magnetic field at a position angle of $\simeq 20^{\circ}$, compared with the $30^{\circ}$ of the galactic plane. Further studies over a wider region (Kobayashi et al., 1983) found that for $\mathrm{H}-\mathrm{K}<1$, corresponding to distances less than $5 \mathrm{kpc}$, the position angle of polarization changed from $20^{\circ}$ to $\simeq 170^{\circ}$ indicating a change of direction of the aligning field near this distance.

Polarized emission in the mid-infrared has often been observed in compact HII regions (this work is being prepared for publication), and grain alignment is expected to be efficient in these conditions (Aitken et al., 1986). Observations of the Galactic Centre beyond $2 \mu \mathrm{m}$ have indicated the presence of both absorptive and emissive components of polarization (Knacke and Capps, 1977; Lebofsky et al., 1982; Bailey et al., 1984; Aitken et al., 1986; Werner et al., 1988). IRS 3, one of the sources prominent at $10 \mu \mathrm{m}$, appears not to be ionized; it has no radio counterpart, shows no recombination lines or ionic fine structure lines, is spatially unresolved and seems optically thick in the middle infrared. It shows no emissive component of polarization and presumably its polarization, showing a strong silicate signature, is due solely to the diffuse material along the line of sight, and representative of the innermost regions where most of the silicate material lies. Here the polarization position angle is $\simeq 0^{\circ}$, and it is found that this angle also gives a more consistent description of the interstellar component in the observations of the ionized filaments which show intrinsic polarization.

Werner et al. (1988) and Hildebrand (1989) report observations of polarized emission at $100 \mu \mathrm{m}$ from dust grains in the $2 \mathrm{pc}$ molecular ring which surrounds the Galactic Centre. The position angle implies an aligning field direction of $\simeq 0^{\circ}$, similar to that observed by absorption at $10 \mu \mathrm{m}$, and it may be that the dust ring is the site of some fraction of the enhanced silicate extinction observed against Sgr A.

Thus it seems that there are (at least) three distinct regions of grain alignment along the path to the Galactic Centre: a "local" region extending to $\simeq 5 \mathrm{kpc}$, a 
further region bearing most of the near infrared extinction, and the central region of enhanced abundance of silicate grains, some of which may arise in the 2 pc molecular ring.

The $10 \mu \mathrm{m}$ spectropolarimetry of IRS 3 (Aitken et al., 1986) is of interest since not only is it the longest, but the only, path in the diffuse ISM for which the polarization signature of silicate material is available. While the spectrum of IRS 3 differs from that of molecular cloud material typified by BN, the spectropolarimetry is unfortunately still of insufficiently high quality to reveal significant differences in polarization; at the one percent polarization error level in IRS 3 the spectral shape of its polarization is indistinguishable from that of $\mathrm{BN}$.

Martin (1975) has shown that the behaviour of the polarization as a function of wavelength through a resonance in the grain material can lead to information on the band strength of the material, a quantity which spectroscopy alone cannot determine. For the BN object Martin showed this to imply band strengths much less than that of laboratory crystalline silicates and more appropriate to amorphous materials. Spectropolarimetry of the Galactic Centre shows that these band strengths $\left(\sim 3000-5000 \mathrm{~cm}^{-1}\right)$ apply also to grains in the diffuse ISM, and in particular to those close to the Galactic Centre (Aitken, 1986).

Polarized emission has been observed in the Galactic Centre from wavelengths as short as $3.5 \mu \mathrm{m}$ (e.g. Bailey et al., 1984) out to $100 \mu \mathrm{m}$ (Werner et al., 1988; Hildebrand, 1989), and the implied range of grain temperatures is from $\sim 50-750$ $K$. It seems almost certain that the grains are magnetically aligned yet, for this to be effective, the grain spin temperatures must be very different from their internal temperatures. In the magnetic relaxation mechanism proposed by Davis and Greenstein (1951), the grains reach rotational equilibrium with the ambient gas, while their internal temperature is controlled by radiation balance and is usually much smaller. However, the hot grains in the ionized filaments suffer efficient electromagnetic damping due to the copious emission of infrared photons (Martin, 1972; Purcell and Spitzer, 1971; Aitken et al., 1986), while the polarized emission at 100 $\mu \mathrm{m}$ arises from the dense neutral ring which surrounds the Centre; in both these cases it appears that the grains must be spinning suprathermally through some variant of the Purcell (1979) pin-wheel mechanism. It also seems necessary that grains which are capable of alignment contain super-paramagnetic (SPM) inclusions (Jones and Spitzer, 1967; Mathis, 1986) in order that the alignment process competes effectively with the disorientating effects of gas collisions and changing surface properties of grains. Finally, the strong and uniform polarized emission in the $10 \mu \mathrm{m}$ region (7-8\%) from the ionized sources in the northern arm suggests that here the alignment may be almost complete; to give the observed polarizations grains need only be slightly asymmetric with aspect ratios $\simeq \mathbf{1 . 2}$.

\section{CONCLUSIONS}

1. The infrared extinction curve along the line of sight to the Galactic Centre is closely similar to that of the diffuse ISM out to $5 \mu \mathrm{m}$, but in the $10 \mu \mathrm{m}$ region the relative strength of silicate absorption is twice that in the local ISM. This reflects the increasing abundance of heavy elements towards the centre of the Galaxy.

2. Apart from absorption features attributed to silicates, only two other weak 
features at 3.0 and $3.4 \mu \mathrm{m}$ are seen, and tentatively ascribed to $O-H$ and $C-H$ bonds respectively. There is no evidence for absorption by the ices which are clearly abundant in molecular clouds.

3. The grains appear to be stratified into at least three distinct regions along the line of sight.

4. The silicate grains along the line of sight have a band strength appropriate to amorphous rather than highly structured material.

5. The strong polarized emission observed from the central regions suggests that grains spin suprathermally, contain super-paramagnetic inclusions, and need not be either very elongated or flattened.

\section{REFERENCES}

Aitken, D. K. 1986, in Light on Dark Matter, Proc. first IRAS Conf., ed. Israel, F. P., (Dordrecht: Reidel), p. 241.

Aitken, D. K., Roche, P. F., Bailey, J. A., Briggs, G. P., Hough, J. H. \& Thomas, J. A. 1986, M. N. R. A. S., $218,363$.

Bailey, J., Hough, J. H. \& Axon, D. J. 1984, M. N. R. A. S., 206, 661.

Becklin, E. E., Mathews, K., Neugebauer, G. \& Willner, S. P. 1978, Ap. J., 219, 121.

Becklin, E. E., Gatley, I. \& Werner, M. N. 1982, Ap. J., 258, 135.

Blanco, V. M. \& McCarthy, M. F. 1981, in Physical Processes in Red Giants, eds. Iben, I. \& Renzini, A., (Dordrecht: Reidel), p. 147-152.

. 1983, A. J., 88, 1442

Brown, R. L., Johnson, K. L. \& Lo, K. Y. 1981. Ap. J., 250, 155.

Butchart, I., McFadzean, A. D., Whittet, D. C. B., Geballe, T. R. \& Greenberg, J. M. 1986, Astr. $A p ., 154$, L5.

Castor, J. I., Simon, T. 1983, Ap. J., 265, 304.

Davidson, Harvey, Lester, Morris \& Werner 1988, in preparation.

Davis, L. \& Greenstein, J. L. 1951 Ap. J., 114, 206.

Duley, W. W. 1988, in Dust in the Universe, eds. Bailey, M. E. \& Williams, D. A., (Cambridge University Press).

Duley, W. W. \& Williams, D. A. 1983, M. N. R. A. S., 205, 67.

Fuenmayor, F. J. 1981, Rev. Mex. Astron. Astrofis., 6, 83.

Gatley, I. 1984, in Proc. Workshop on Infrared Spectra of Interstellar Dust, Occasional Reports of the Royal Observatory, Edinburgh: no. 12, p. 118.

Gillett, F. C., Jones, T. W., Merrill, K. M. \& Stein, W. A. 1975, Astr. Ap., 45, 77.

Glass, I., Catchpole, R. M. \& Whitelock, P. A. 1987, M. N. R. A. S., 227, 373.

Greenberg, J. M. \& Hong, S. S. 1974, in HII Regions and the Galactic Centre, VIII ESLAB Symp., ed. Moorwood, (A.F.M.), 221.

Hildebrand, R. H. 1989, in IAU Symposium 195: Interstellar Dust, eds. L. J. Allamandola and A. G. G. M. Tielens, (Dordrecht: Reidel), p. 275.

Jones, R. V. \& Spitzer, L. 1967, Ap. J., 147, 943.

Kobayashi, Y., Kawara, K., Kozasa, T., Sato, S. \& Okuda, H. 1980, Pub. Astr. Soc. Japan, 32, 291.

Kobayashi, Y., Okuda, H., Sato, S., Jugaku, J. \& Dyck, H. M. 1983, Pub. Astr. Soc. Japan, \$5, 101.

Knacke, R. F. \& Capps, R. W. 1977, Ap. J., 261, 271.

Lebofsky, M. J., Rieke, G. H., Deshpande, M. R. \& Kemp, J. C. 1982, Ap. J., $268,672$.

Lebofsky, M. J. \& Rieke, G. H. 1987, in The Galactic Center AIP Conf. Proc. 155, ed. Backer, D. C., (New York: A.I.P.), p. 79.

Leitherer, C. \& Wolf, B. 1984, Astr. Ap., 132, 151.

Lester, D. F., Joy, M., Harvey, P. M. \& Ellis, H. B. 1987, The Galactic Center AIP Conf. Proc. 155, ed. Backer, D.C., (New York: A.I.P.), p. 138. 
McFadzean, A. D., Longmore, A. J., Bode, M. F., Whittet, D. C. B. \& Adamson, A. J. 1988, in preparation for $M$. N. R. A.S..

Martin, P. G. 1972, M. N. R. A. S., 158, 63.

. 1975, Ap. J., 202, 393.

Mathis, J. S. 1986, Ap. J., 308, 281

Purcell, E. M. 1979, Ap. J., 231, 404.

Purcell, E. M. \& Spitzer, L. 1971, Ap. J., 167, 31.

Rieke, G. H. 1974, Ap. J., 198, 181.

Rieke, G. H. \& Lebofsky, M. J. 1985, Ap. J., 288, 618.

Roche, P. F. 1988, in Dust in the Universe, eds. Bailey, M. E. \& Williams, D. A., (Cambridge University Press).

Roche, P. F. 1989, in IAU Symposium 195: Interstellar Dust, eds. L. J. Allamandola and A. G. G. M. Tielens, (Dordrecht: Kluwer), p. 303.

Roche, P. F. \& Aitken, D. K. 1984, M. N. R. A. S., 208, 481.

. 1985, M. N.R.A.S., 215, 425.

Savage, B. D. \& Mathis, J.'S. 1979, Ann. Rev. Astr. Ap., 17, 73.

Tapia et al. 1989, in Interstellar Dust Contributed Papers, eds. A. G. G. M. Tielens and Allamandola, L. J., NASA CP-3036.

Wade, R., Geballe, T. R., Krisciunas, K., Gatley, I. \& Bird, M. C. 1987, Ap. J., \$20, 570.

Werner, M. W., Davidson, J. A., Hildebrand, R. H., Morris, M. R., Novak, G. \& Platt, S. R. 1988, submitted Ap. J..

Whittet, D. C. B. 1988, in Dust in the Universe, eds. Bailey, M. E. \& Williams, D. A., (Cambridge University Press).

Whittet, D. C. B. \& van Breda 1980, M. N. R. A. S., 192, 467.

Willner, S. P. 1984, in Galactic and Extragalactic Infrared Spectroscopy XVI ESLAB Symp., ed. Moorwood, (A.F.M.). p. 37.

Willner, S. P. \& Pipher, J. L. 1982, in The Galactic Center AIP Conf. Proc. 88, eds. Riegler, G. R. \& Blandford, R. D., p. 77. 


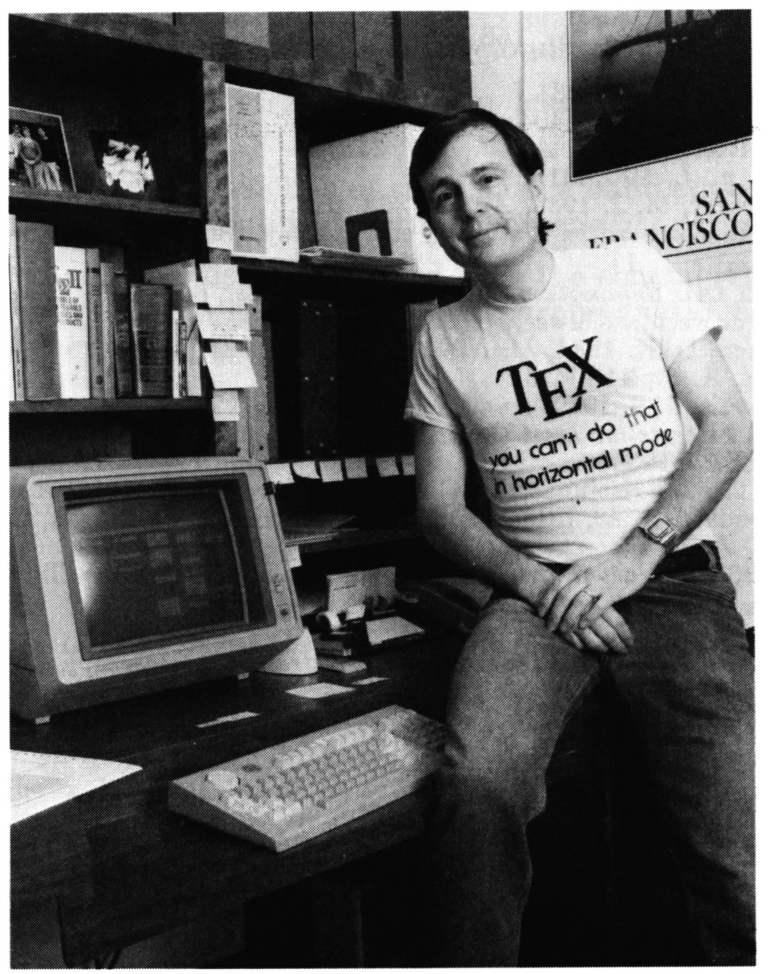

Gary Villere, $\mathrm{T}_{\mathrm{E} X n i c i a n}$ 